Volume 8, No.1.4, 2019

International Journal of Advanced Trends in Computer Science and Engineering

Available Online at http://www.warse.org/IJATCSE/static/pdf/file/ijatcse3181.42019.pdf

https://doi.org/10.30534/ijatcse/2019/3181.42019

\title{
Augmentation of NavIC with GPS Over Indian Region
}

\author{
Sudershan Reddy Kotla ${ }^{1}$, Sameeha Fahmeen ${ }^{2}$, Quddusa Sultana ${ }^{3}$, D Krishna Reddy ${ }^{4}$ \\ ${ }^{1}$ Department of Electronics and Communication Engineering, Chaitanya Bharati Institute of Technology, India \\ kotla66@yahoo.com \\ ${ }^{2}$ Department of Computer Science Engineering, Shadan Women's College of Engineering and Technology, India \\ samfahmeen08@gmail.com \\ ${ }^{3}$ Department of Electronics and Communication Engineering, Deccan College of Engineering and Technology \\ India, quddusas@gmail.com \\ ${ }^{4}$ Department of Electronics and Communication, Chaitanya Bharati Institute of Technology, India \\ dkreddy@cbit.ac.in
}

\begin{abstract}
Global Positioning System (GPS) satellites are used to provide navigational services to the users in India. But now for security reasons, Indian Space Research Organization (ISRO) has developed its own navigation satellite system called as Indian Regional Navigation Satellite System (IRNSS). IRNSS is renamed as Navigation with Indian Constellation (NavIC). NavIC is an emerging satellite based navigation system offering an independent positioning and timing service over India and neighboring regions. Position accuracy of NavIC is $10 \mathrm{~m}$ on land and $20 \mathrm{~m}$ in Indian Ocean within $1500 \mathrm{~km}$ around Indian Boundary. Moreover, to increase user position accuracy, the NavIC can be augmented with other navigation systems. This paper focuses, initially, on the analysis of satellite visibility of augmented NavIC with GPS. Comparative analysis of NavIC and NavIC augmented with GPS is also done in terms of Position Dilution of Precision (PDOP). PDOP specifies the user position error caused by the relative position of the satellites.
\end{abstract}

Key words: DOP, GPS, NavIC, Satellite Visibility.

\section{INTRODUCTION}

In May 2006, India decided to develop its own Navigation satellite system called as Indian Regional Navigation Satellite System (IRNSS) [1]. IRNSS is also known as Navigation with Indian Constellation (NavIC). The requirement of such a navigation system is driven by the fact that access to Global Navigation Satellite Systems (GNSSs) like GPS is not guaranteed in hostile situations. NavIC provides two services, one is the Standard Positioning Service (SPS) open for civil use and the other is Restricted Service (RS), encrypted one, for authorized users (military). NavIC if augmented with other navigation systems is expected to provide navigation and guidance with good accuracy. This paper focuses on the assessment of satellite visibility of NavIC-7 (NaivIC with 7 satellits) augmented with GPS. Comparative analysis of the augmented system with standalone NavIC-7 is also done in terms of PDOP.

\section{OVERVIEW OF GPS AND NAVIC}

The GPS constellation consists of a minimum of 24 satellites positioned in six orbital planes. Each orbit consists of 4 satellites. The orbital planes are inclined at an angle of $55^{\circ}$ with respect to the equator. A minimum of 4 satellites are visible from any point on the surface of the earth. The GPS satellites are placed at a height of 20,200 km from the surface of the earth[2].

The NavIC has three segments. They are: Space segment, Ground segment and User segment. The NavIC space segment has a constellation of 7 satellites, orbiting above the earth at a height of $36000 \mathrm{~km}$ approximately. Out of 7 satellites, 3 satellites are placed in Geostationary Orbit (GEO) and 4 satellites are placed in Geosynchronous Orbits (GSO). NavIC satellites in Geosynchronous Orbits (GSO) are at a height of $36000 \mathrm{~km}$, and are inclined at an angle of $\pm 29^{\circ}$ with the equator [3]. Due to this inclination, satellites provide coverage to the higher and lower latitudes near the poles. In Geostationary Orbit (GEO) they remain above the equator.

\section{ESTIMATION OF SATELLITE VISIBILITY OF NAVIC-7 AUGMENTED WITH GPS}

In case of NavIC-7, three SVs (IRNSS-1C, 1F, 1G) are GEO and four SVs (IRNSS-1I, 1B, 1D, 1E) are GSO. There is a possibility of the overlap of two GSO SVs (IRNSS-1I,1D) with the other two GSO SVs (IRNSS-1B,1E) respectively, twice a day, deteriorating the geometry required for proper positioning. Hence, the best as well as the worst cases of satellite visibility are considered for NavIC-7. Augmentation of NavIC with GPS is done according to their respective timings i.e. the constellation of NavIC at 6:00 am is augmented with the constellation of GPS at the same time and so on [4]. Latitude and longitude range of satellite visibility for all the four cases is shown in Table 1. 


\subsection{NavIC-7 Augmented with GPS at 6:00am (Case-1):}

Satellite visibility of GPS augmented with NavIC-7 at 6:00am (Case-1) over the Indian region is shown in Figure 1. It can be observed that around $10^{\circ} \mathrm{N}-25^{\circ} \mathrm{N}$ and $77^{\circ} \mathrm{E}-93^{\circ} \mathrm{E}$ number of SVs are maximum i.e., 12. A minimum of 6 satellites can also be observed over a wide range of latitudes $\left(45^{\circ} \mathrm{N}-0^{\circ}\right)$ and longitudes $\left(60^{\circ} \mathrm{E}-100^{\circ} \mathrm{E}\right)$.

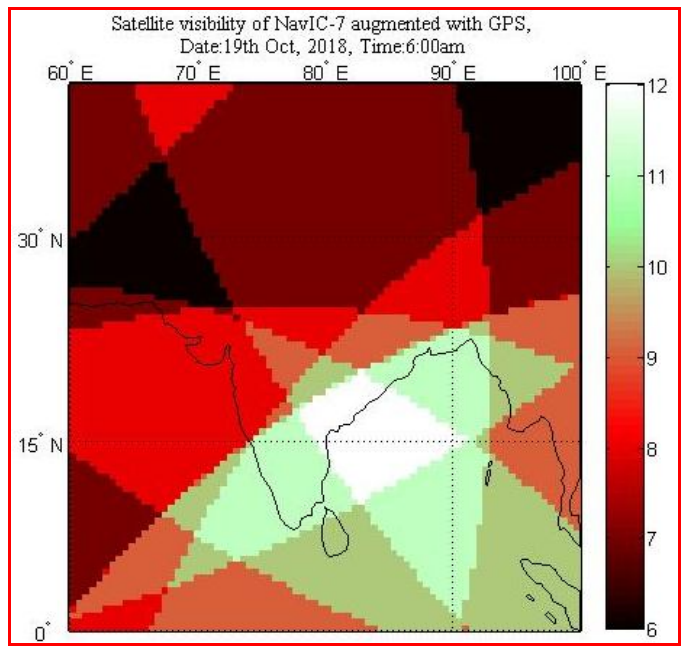

Figure 1: Satellite visibility of NavIC-7 augmented with GPS at 6:00am (Case-1)

\subsection{NavIC-7 Augmented with GPS at 6:00pm (Case-2):}

Satellite visibility of NavIC-7 augmented with GPS at 6:00pm (Case-2) over the Indian region is shown in Figure 2. It can be observed that around $15^{\circ} \mathrm{N}$ to $7^{\circ} \mathrm{N}$ and around $75^{\circ} \mathrm{E}$ number of SVs are maximum i.e., 14. A minimum of 7 satellites can also be observed over a wide range of latitudes $\left(0^{\circ}-60^{\circ} \mathrm{N}\right)$ and longitudes $\left(60^{\circ} \mathrm{E}-100^{\circ} \mathrm{E}\right)$.

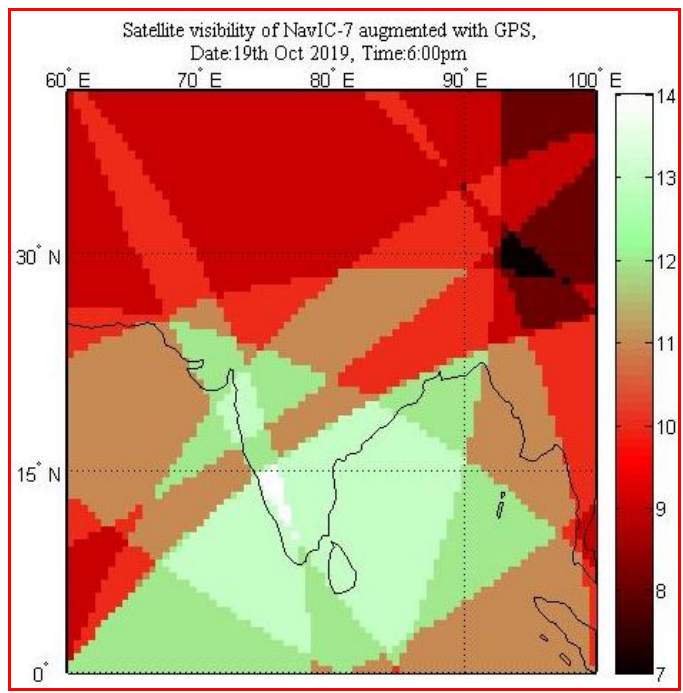

Figure 2: Satellite visibility of NavIC-7 augmented with GPS at 6:00pm (Case-2)

\subsection{NavIC-7 Augmented with GPS at 12:00am (Case-3):}

Satellite visibility of NavIC-7 augmented with GPS at 12:00am (Case-3) over the Indian Region is shown in Figure 3. A maximum of 16 satellites are present in between $73^{\circ} \mathrm{E}$ to $87^{\circ} \mathrm{E}$ and $5^{\circ} \mathrm{N}$ to $19^{\circ} \mathrm{N}$ (approximately).13-16 SVs are found over Indian region.

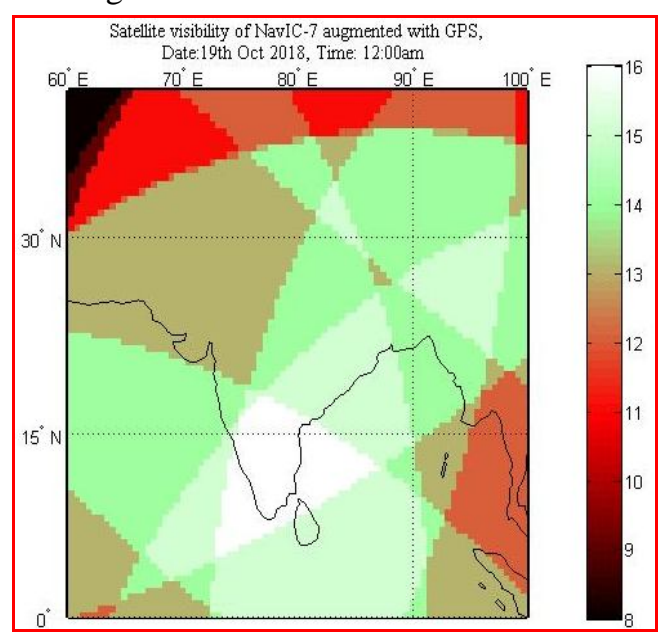

Figure 3: Satellite visibility of NavIC-7 augmented with GPS at 12:00am (Case-3)

\subsection{NavIC-7 Augmented with GPS at 12:00pm (Case-4):}

Satellite visibility of NavIC-7 augmented with GPS at 12:00pm (Case-4) over the Indian region is shown in Figure 4. A maximum of 13 satellites are found in between $60^{\circ} \mathrm{E}$ to $83^{\circ} \mathrm{E}$ longitudes and, $0^{\circ}$ to $8^{\circ} \mathrm{N}$ (approximately). The results are summarized in Table 1.

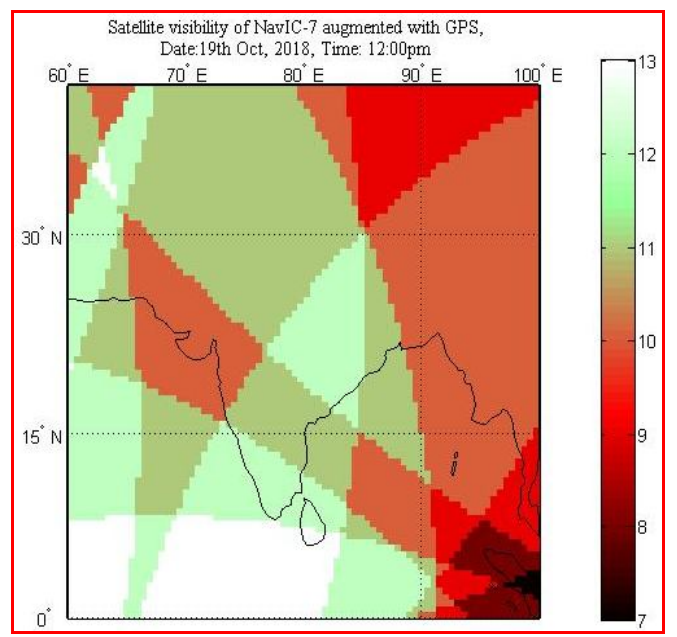

Figure 4: Satellite visibility of NavIC-7 augmented with GPS at 12:00pm (Case-4) 
Table 1: Satellite visibility of NavIC-7 augmented with GPS

\begin{tabular}{|c|c|c|c|}
\hline NavIC-7+GPS & $\begin{array}{c}\text { No. of } \\
\text { SVs } \\
\text { visible }\end{array}$ & $\begin{array}{c}\text { Latitude } \\
\text { range }\end{array}$ & $\begin{array}{c}\text { Longitude } \\
\text { range }\end{array}$ \\
\hline Case-1 (6:00am) & 12 & $10^{\circ} \mathrm{N}-25^{\circ} \mathrm{N}$ & $77^{\circ} \mathrm{E}-93 \mathrm{E}^{\mathrm{o}}$ \\
\hline Case-2 (6:00pm) & 14 & $15^{\circ} \mathrm{N}-7^{\circ} \mathrm{N}$ & Around $75^{\circ} \mathrm{E}$ \\
\hline Case-3 (12:00am) & 16 & $5^{\circ} \mathrm{N}-19^{\circ} \mathrm{N}$ & $73^{\circ} \mathrm{E}-87 \mathrm{E}^{\mathrm{o}}$ \\
\hline Case-4 (12:00pm) & 13 & $0^{\circ}-8^{\circ} \mathrm{N}$ & $60^{\circ} \mathrm{E}-83^{\circ} \mathrm{E}$ \\
\hline
\end{tabular}

\section{ESTIMATION OF PDOP FOR NAVIC-7 AUGMENTED WITH GPS}

PDOP values are computed and analyzed for the best and worst cases of NavIC-7 augmented with GPS (6:00am, 6:00pm, 12:00am and 12:00pm) respectively [5]. For all the results, Indian subcontinent with latitude range of $0^{\circ}$ to $40^{\circ} \mathrm{N}$ and longitude range of $60^{\circ} \mathrm{E}$ to $100^{\circ} \mathrm{E}$ is considered for better focus and comparative analysis [6].

PDOP values for NavIC-7 augmented with GPS at 6:00am (Case-1) are shown in Figure 5.The minimum DOP values are observed over southern part of India. Minimum PDOP is 1.41. The maximum values of DOP are observed over the north-west of India. The maximum PDOP is 19.89.

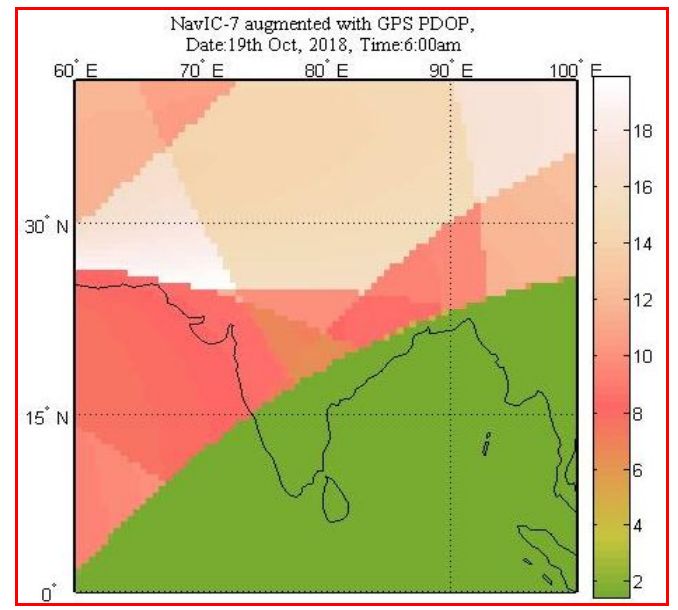

Figure 5: PDOP of NavIC-7augmented with GPS at 6:00am (Case-1)

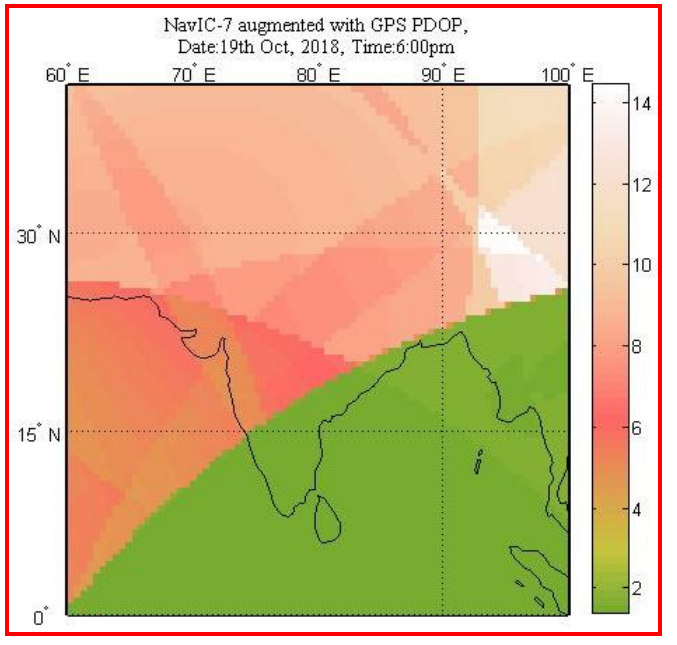

Figure 6: PDOP of NavIC-7augmented with GPS at 6:00pm

(Case-2)

The PDOP values for NavIC-7 augmented with GPS at 6:00pm (Case-2) are shown in Figure 6 respectively. The minimum DOP values are observed over the southern part of India. Minimum PDOP is 1.39. The maximum values of DOP are observed over the south and eastern coast of India. The maximum PDOP is 14.45 .

The PDOP values for NavIC-7 augmented with GPS at 12:00am (Case-3) are estimated and plotted as shown in Figure 7 respectively. The minimum DOP values are observed over southern part of India. Minimum PDOP is 1.38. The maximum values of DOP are observed over the north-west part of India. The maximum PDOP is 10.17.

The PDOP values for NavIC-7 augmented with GPS at $12: 00 \mathrm{pm}$ (Case-4) are estimated and plotted as shown in Figure 8. The minimum DOP values are observed over southern part of India. Minimum PDOP is 1.42. The maximum values of DOP are observed over the north-west part of India. The maximum PDOP is 10.09 .

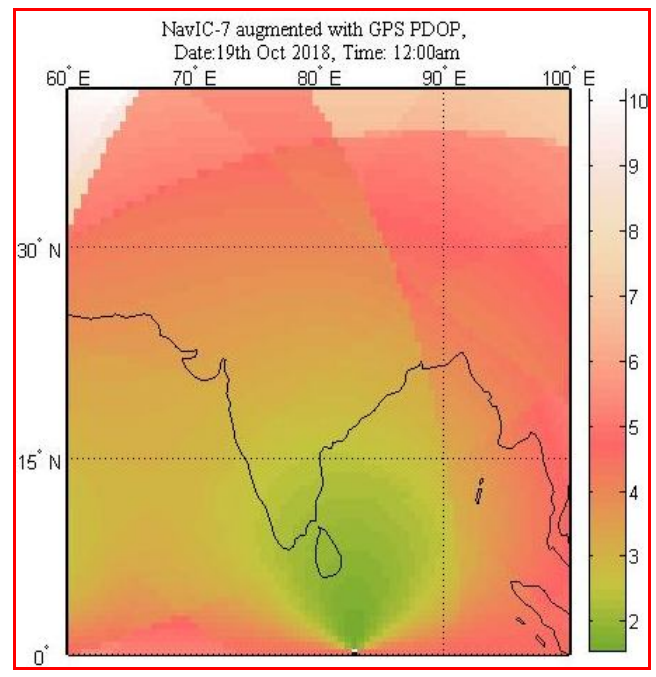

Figure 7: PDOP of NavIC-7augmented with GPS at 12:00am (Case-3) 


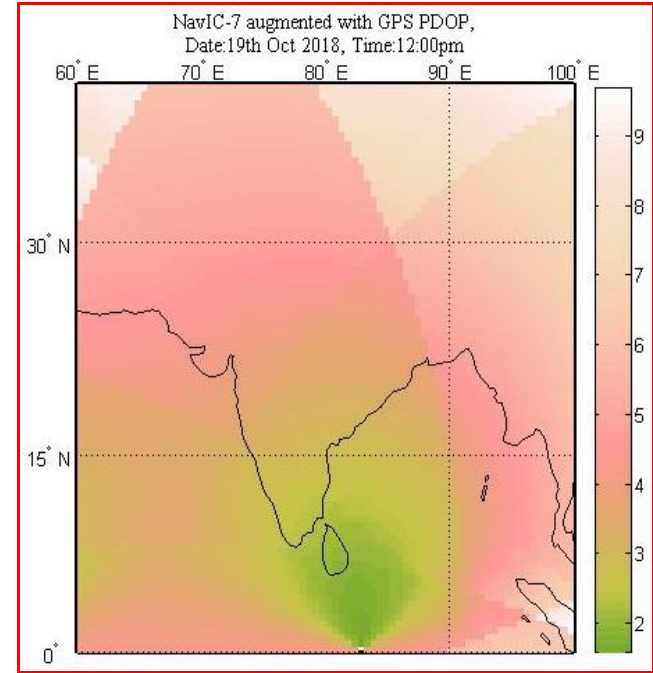

Figure 8: PDOP of NavIC-7augmented with GPS at 12:00pm (Case-4)

Minimum, Maximum and mean values of PDOP for NavIC-7 augmented with GPS and standalone NavIC-7 are shown in Table 2 and 3 respectively. Comparing the PDOP values of standalone NavIC with NavIC-7 augmented with GPS, it can be observed that for case- 1 and case- 2 minimum PDOP of standalone NavIC is 3.02. For NavIC-7 augmented with GPS (Case-1 and Case-2) minimum PDOP is 1.41 and 1.39 respectively. For case-3 and case-4 the PDOP for standalone NavIC is infinity which is not applicable for any navigation system. For NavIC-7 augmented with GPS (Case-3 and Case-4) it is 1.38 and 1.42 respectively.

Table 2: $\quad$ PDOP for NavIC-7 augmented with GPS

\begin{tabular}{|l|c|c|c|c|c|c|}
\hline \multicolumn{7}{|c|}{ PDOP of NavIC-7 augmented with GPS } \\
\hline & \multicolumn{3}{|c|}{ (Case-1, 6:00am) } & \multicolumn{3}{c|}{ (Case-2, 6:00pm) } \\
\hline DOP & Min & Max & Mean & Min & Max & Mean \\
\hline PDOP & 1.41 & 19.89 & 10.65 & 1.39 & 14.45 & 7.92 \\
\hline & \multicolumn{3}{|c|}{ (Case-3, 12:00am) } & \multicolumn{3}{c|}{ (Case-4, 12:00pm) } \\
\hline PDOP & 1.38 & 10.17 & 5.77 & 1.42 & 10.09 & 5.74 \\
\hline
\end{tabular}

Table 3: $\quad$ PDOP for standalone NavIC-7

\begin{tabular}{|c|c|c|}
\hline \multicolumn{3}{|c|}{ PDOP for standalone NavIC } \\
\hline \multicolumn{2}{|c|}{ (Case-1 (6:00am)/Case-2 (6:00pm)) } \\
\hline Min & Max & Mean \\
\hline 3.02 & 6.18 & 4.43 \\
\hline (Case-3 (12:00am)/Case-4 (12:00pm)) \\
\hline Min & Max & Mean \\
\hline Infinity & Infinity & Infinity \\
\hline
\end{tabular}

\section{CONCLUSION}

Satellite visibility is an important parameter to be considered while developing a navigation system. This paper presents the satellite visibility of NavIC augmented with GPS. PDOP values are also computed for NavIC augmented with GPS and for standalone GPS. It can be observed that PDOP values decreases as the number of satellites increases. For instance minimum PDOP value of standalone NavIC at 6:00 am and 6:00 pm is 3.02 where as for NavIC augmented with GPS it is 1.41 and 1.38. NavIC augmented with GPS show better PDOP compared to standalone NavIC.

\section{REFERENCES}

1. www.ISRO.gov.in

2. Misra $P$ and Per Enge, "Global Positioning System-Signals, Measurements and Performance", Ganga-Jamuna Press, Lincoln,Massachusetts, USA, 2001.

3. Parimal Majithiya, Kriti Khatri, S. C.Bera, S. Sarkar, K.S Parikh, "Future Space Service of NavIC (IRNSS) Constellation", Inside GNSS, July/August 2017.

4. K Sudershan Reddy, Sameeha Fahmeen, Quddusa Sultana, D Krishna Reddy, "Augmentation of NavIC with BeiDou-2 over India Region", "The International Workshop on Antenna Technology”, IEEE, Miami, USA, $3^{\text {rd }}-6^{\text {th }}$ March, 2019.

5. I. S. Jacobs and C. P. Bean, "Fine particles, thin films and exchange anisotropy," in Magnetism, vol. III, G. T. Rado and H. Suhl, Eds. New York: Academic, 1963, pp. 271-350.

https://doi.org/10.1016/B978-0-12-575303-6.50013-0

6. Achanta D Sarma, Quddusa Sultana, Vemuri Satya Srinivas, "Augmentation of Indian Regional Navigation Satellite System to Improve Dilution of Precision", The Journal of Navigation, Volume 63, Issue 2, April 2010, pp. 313-321.

https://doi.org/10.1017/S037346330999035X 